\title{
The Inhibition of Methane Hydrate Formation by Water Alignment underneath Surface Adsorption of Surfactants
}

\author{
Ngoc N. Nguyen ${ }^{(a)}$, Anh V. Nguyen ${ }^{(a), *}$, Liem X. Dang ${ }^{(b)}$ \\ ${ }^{(a)}$ School of Chemical Engineering, The University of Queensland, Brisbane, QLD 4072, Australia \\ ${ }^{(b)}$ Fundamental and Computational Sciences Directorate, Pacific Northwest National Laboratory, \\ Richland, WA 99352, United States \\ *Corresponding author. Tel.: +61 7336 53665; Fax: +61 7336 54199; \\ Email: anh.nguyen@eng.uq.edu.au
}

\begin{abstract}
Sodium dodecyl sulfate (SDS) has been widely shown to strongly promote the formation of methane hydrate. Here we show that SDS displays an extraordinary inhibition effect on methane hydrate formation when the surfactant is used in sub-millimolar concentration (around $0.3 \mathrm{mM}$ ). We have also employed Sum Frequency Generation vibrational spectroscopy (SFG) and molecular dynamics simulation (MDS) to elucidate the molecular mechanism of this inhibition. The SFG and MDS results revealed a strong alignment of water molecules underneath surface adsorption of SDS in its sub-millimolar solution. Interestingly, both the alignment of water and the inhibition effect (in $0.3 \mathrm{mM}$ SDS solution) went vanishing when an oppositely-charged surfactant (tetra-n-butylammonium bromide, TBAB) was suitably added to produce a mixed solution of $0.3 \mathrm{mM}$ SDS and $3.6 \mathrm{mM}$ TBAB. Combining structural and kinetic results, we pointed out that the alignment of water underneath surface adsorption of dodecyl sulfate (DS ${ }^{-}$) anions gave rise to the unexpected inhibition of methane hydration formation in sub-millimolar solution of SDS. The adoption of TBAB mitigated the SDS-induced electrostatic field at the solution's surface and, therefore, weakened the alignment of interfacial water which, in turn, erased the inhibition effect. We discussed this finding using the concept of activation energy of the interfacial formation of gas hydrate. The main finding of this work is to reveal the interplay of interfacial water in governing gas hydrate formation which sheds light on a universal molecular-scale understanding of the influence of surfactants on gas hydrate formation.
\end{abstract}


Keywords: gas hydrate, methane, surfactant, water structure

Graphic Abstract

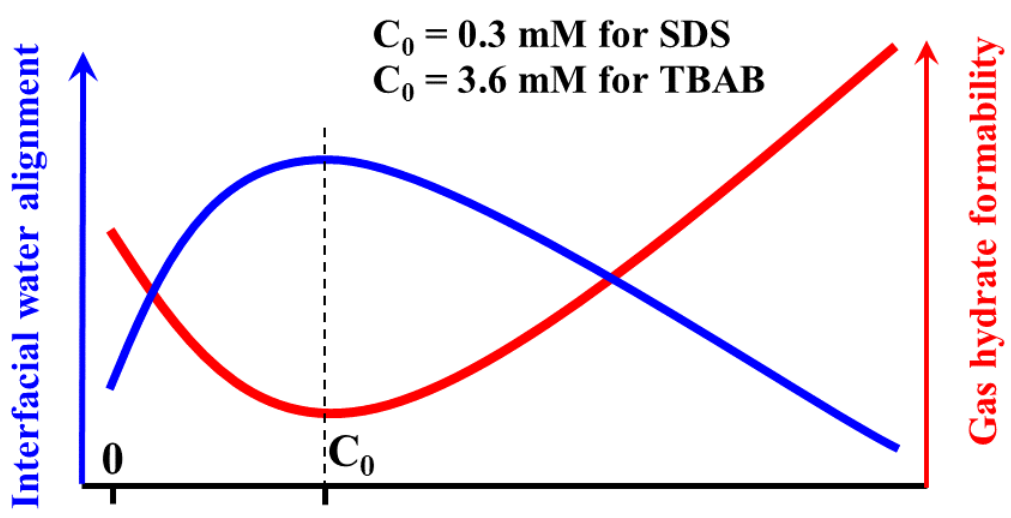

Concentration of surfactant

\section{INTRODUCTION}

Gas hydrates are ice-like crystalline solids comprising water and suitable gases (e.g. $\mathrm{CH}_{4}$, $\mathrm{CO}_{2}$, etc.). The water molecules (the host) form a cage-like hydrogen bonded network which encapsulates the gas molecules (the guest) inside, and the encaged gas molecules, in turn, exert a multi-directional force to prevent the cage-like structure from collapsing ${ }^{1-2}$. Despite such astounding simplicity of their chemical composition, the science of gas hydrates is fascinating and the potential applications are tremendous. For example, the formation of gas hydrates inside submarine pipelines is a well-known hazard to offshore gas/oil exploitation ${ }^{3-4}$ whereas the worldwide abundance of natural (methane) hydrates provides excellent energy sources ${ }^{5-8}$. Especially, artificial gas hydrates can open up novel methods for gas storage and transportation ${ }^{9-}$ ${ }^{12}$, gas separation ${ }^{13-20}$ as well as carbon dioxide sequestration ${ }^{21-24}$. However, in all cases, the formation of gas hydrates needs to be controlled in a desired manner, either to inhibit the formation if the resulting hydrate is unexpected or to promote it if the resulting hydrate is beneficial.

The use of surfactants is one of the most viable ways to control gas hydrate formation owing to their strong effects on gas hydrate kinetics and thermodynamics ${ }^{25-35}$. Amongst the 
surfactants investigated, sodium dodecyl sulfate (SDS) has gained the most attention of researchers due to its high effectiveness in promoting gas hydrate formation 25-26, 30-31. Nonetheless, the fundamentals of experimental observations remain unclear and debatable. Previous works mainly focused on macroscopic measurements of gas hydrate formation. However, the results from those works are experiment-specific, meaning that the experimental observations depend on the conditions adopted (e.g. the concentrations and equipment type, etc.). For example, while SDS is widely known an effective promoter, Sowa et al. ${ }^{36}$ recently reported that this surfactant could act as an inhibitor of gas hydrate formation in a wide range of experimental condition. Similarly, we have shown in our recent work ${ }^{37}$ that tetra-n-butyl ammonium bromide (TBAB), also a well-known gas hydrate promoter, could display a strong inhibiting effect on $\mathrm{CO}_{2}$ gas hydrate formation when it was used at millimolar concentration. Although the explanations of the promoting effect of surfactants have relied on the formation of micelles which can assist the gas dissolution and seeding gas hydrate nucleation 26,38 , the inhibiting effect has not been understood at a molecular scale.

In the aspect of fundamentals, the aforementioned context reflects a lack of a universal understanding of the microscopic pathway of surfactant effects, especially in low concentration regime. Indeed, the fact that current literature neglects the importance of interfacial water when discussing the influence of surfactants (on gas hydrate formation) is of major shortcoming because the interface between gas and water has been observed to be the starting points of gas hydrate nucleation ${ }^{39-41}$. In other words, local water molecules at gas-water interface involve directly in the nucleation of gas hydrate whereby they undergo structural rearrangement and transformation. From this standpoint, it is required to know the surfactant-induced changes in the interfacial water in order to understand how the surfactant affects the subsequent formation of gas hydrate in the according condition. Previous works were not able to address such requirement because the instrumental techniques employed in gas hydrate research so far were not interface-specific, and hence, were unable to differentiate the properties of the interfacial water from the bulk phase.

The aim of this work is, therefore, to gain molecular insights into the effect of SDS on methane hydrate formation. We carried out a systematic study on the effects of SDS, of a wide range of concentration, on gas hydrate kinetics. We also used surface-specific Sum frequency 
generation (SFG) vibrational spectroscopy to probe and analyze the properties of the interfacial water at methane-solution interfaces. Although SFG has become a powerful tool for interface probes, it has not been used in gas hydrate-related research. In the meantime, we employed molecular dynamics simulation (MDS) to study the surface adsorption of SDS and the induced change in the water structure underneath adsorption layer. The outcome of this work has shed light into the interplay of the interfacial water in governing methane hydrate formation and advances our universal understanding of the molecular mechanism underlying of gas hydrate inhibition/promotion by surfactants.

\section{EXPERIMENTAL METHODS}

\subsection{Materials}

Chemicals used in this study included methane (99.995\%, Coregas, Australia), Sodium dodecyl sulfate (99\%, Sigma-Aldrich, USA) and Tetra-n-butylammonium bromide (98\%, Wako, Japan). Deionised water used in this work was produced by a Milli-Q purification system (Milipore, USA).

\subsection{Gas hydrate formation experiment}

Gas hydrate kinetics experiments were carried out using a typical experimental setup for gas hydrate synthesis, which consists of a high-pressure reactor (Parr Instruments, USA), a cooling system and gas supply system. The instantaneous temperature and pressure inside the reactor were simultaneously monitored and recorded by a data acquisition system. A fuller description of the experimental procedure has been reported in our previous publication ${ }^{42-43}$. The gas uptake was calculated using state equation of real gas in which the compressibility factor of methane was calculated by using Brill-Beggs correlation ${ }^{44}$. The details of the calculation method are presented elsewhere ${ }^{42}$.

\subsection{SFG experiment}

Sum Frequency Generation (SFG) vibrational spectroscopy measurements were carried out on EKSPLA SFG system (EKSPLA, Lithuania). The visible beam and the tunable IR beam were overlapped spatially and temporally on the methane-solution interface. The visible beam was 
generated by frequency doubling the fundamental output pulses $(1064 \mathrm{~nm}, 10 \mathrm{~Hz})$ of 20 ps pulsewidth from an EKSPLA solid state Nd:YAG laser. The tunable IR beam was generated from an EKSPLA optical parametric generation/amplification and difference frequency system based on LBO and $\mathrm{AgGaS}_{2}$ crystals. SFG measurements were carried out using ssp polarization combination of the output and input beams (s-polarized SFG, s-polarized visible and p-polarized IR) with the incident angle of the visible beam set to $\alpha_{v i s}=60^{\circ}$ and that of the IR set to $\alpha_{I R}=54^{o}$. The signal was fine-tuned $3200 \mathrm{~cm}^{-1}$ for $3000-3600 \mathrm{~cm}^{-1}$ region.

Figure 1 schematically shows the setup of sampling system for probing methane-solution interface. Methane was charged into the sampling cell prior to the measurement. The sampling cell consists of a cap (1) and a base (2). The cap has two transparent $\mathrm{CaF}_{2}$ crystal windows (5) which allow the beams to travel through. The cell was also assembled to an external mechanical rig (not shown) for adjusting the configuration. Even though the SFG experiments were only performed under ambient pressure due to the specifications of the instrument, the results would be applicable to the case of high pressure because surface adsorption of surfactant always occurs.

For the interpretation of the spectra, the experimentally measured signals were fitted with theoretical modulus model of SFG spectroscopy (see Supporting Information).

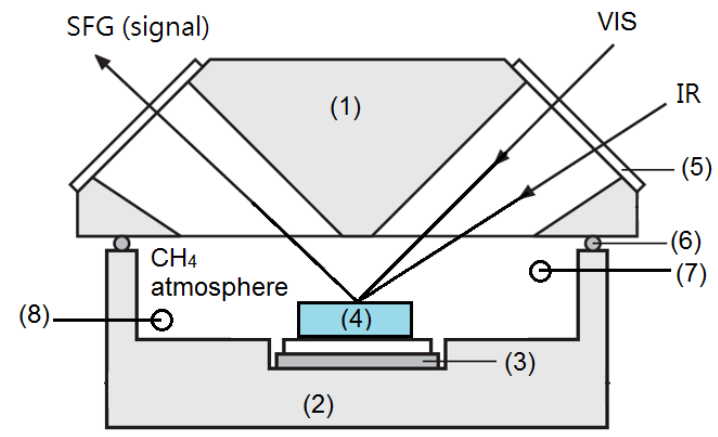

Figure 1. Sampling system for recording SFG signal for methane-solution interface, consisting of: (1): cell cap, (2): cell base, (3): sample base, (4): solution, (5): transparent $\mathrm{CaF}_{2}$ crystal windows, (6): O-ring, (7) and (8): inlet and outlet of methane stream. 


\section{SIMULATION}

The molecular models were created using AmberTools14, and molecular dynamics simulations were performed in Amber9 software ${ }^{45-46}$. TIP4P-ew was used for water model ${ }^{47}$. SDS molecule was modeled using the all-atoms carbon model with hydrogen atoms being explicitly described ${ }^{48}$. The molecular parameters (bonds, angles, and torsions) were retrieved from General Amber Force Field (GAFF) and literature ${ }^{49-50}$. Simulation systems were created by inserting molecules into simulation box, using Packmol software ${ }^{51}$. To simulate methanesolution interface, the simulation box (an orthogonal box with initial $x, y, z$ being 50, 50, $100 \AA$, respectively) was divided into three spatial zones on the $z$-axis. $\mathrm{H}_{2} \mathrm{O}$ and SDS were inserted into $15 \AA \leq z \leq 85 \AA$ zone and formed an aqueous solution while methane occupied the rest of the box. This configuration produced two methane-solution interfaces at $z_{\text {low }}=15 \AA$ and $z_{\text {high }}=$ $85 \AA$, respectively. The number of SDS molecules in simulation boxes was 10, 15 and 30 corresponding to its total concentration being $0.095 \mathrm{M}, 0.190 \mathrm{M}$ and $0.285 \mathrm{M}$, respectively. For convenience, these systems are called System A, B and C, respectively.

For each simulation, after running energy minimization, molecular dynamics was performed for 60 (ns) with the time step of 2 (fs). Simulations were run with NPT ensemble with $T=274 \mathrm{~K}$ and $P=7.5 \mathrm{MPa}$. During simulation, the temperature coupling was controlled using the Langevin dynamics scheme and the pressure scaling was controlled using the Berendsen barostat. The cutoff radius of Lennard-Jones interactions was $11 \AA$. The Ewald summation technique was used to handle long-range electrostatic interactions and the SHAKE algorithm to fix the internal molecular geometry ${ }^{52}$. Simulations were performed using computational resources at Pacific Northwest National Laboratory, Richland, USA.

From the molecular trajectories as the outputs of simulations, we calculated the orientational parameter, $\langle\cos \varphi\rangle$, of water molecules along $z$-axis. For the calculation, the simulation box was divided into different slabs in parallel with the $x y$ plane, and each slab has a thickness of $1 \AA$. Then, $\langle\cos \varphi\rangle$ was calculated using Equation (1) where $\varphi$ is the angle between the $z$-axis and dipole vector of the water molecule; $N_{\text {water }}$ is the number of water molecules in the current slab and $\Delta z$ is the distance from the current slab to the Gibbs dividing surface. Figure 2 gives a schematic illustration of the angle $\varphi$ in this work. 


$$
\langle\cos \varphi\rangle_{\Delta z}=\left\langle\frac{\sum \cos \varphi}{N_{\text {water }}}\right\rangle_{\Delta z}
$$

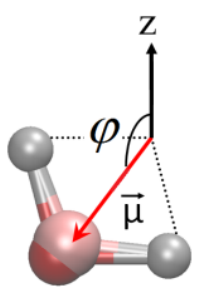

Figure 2. The definition of $\varphi$ in this work.

\section{RESULTS AND DISCUSSIONS}

\subsection{Effect of SDS on gas hydrate formation kinetics}

T-P graphs: Figure 3 shows a typical change in the temperature $(\mathrm{T})$ and pressure $(\mathrm{P})$ versus time recorded from the hydrate reactor. Typically, the duration of each experiment can be divided into four distinct periods. The first stage encompassed the first several minutes of the experiment course in which both $\mathrm{P}$ and $\mathrm{T}$ dropped sharply. The drops in pressure and temperature in this stage were mainly induced by the gas contraction and gas dissolution due to cooling (when the reactor was being submerged into the cooling bath). After a few minutes, the temperature remained well constant at around $0.5^{\circ} \mathrm{C}$, indicating that the cooling period had finished and was followed by an induction period. In the induction stage, the temperature remained well constant, while the pressure continued to decrease steadily, indicating the ongoing dissolution of methane into water. This awaiting period was for the creation of a supersaturated gas in water which was needed for gas hydrate nucleation. Also during this time, water

molecules rearranged to accommodate the gas and transformed into a pre-clathrate-like conformation which could be considered as the evolution of gas hydrate nucleation. The duration of this period (called the induction time) varied greatly with the change in experiment condition. After that, growth stage was following. Apparently, the initiation of growth stage was signified by a catastrophic drop in the pressure along with a sharp rise in the temperature, both due to the exothermic formation gas hydrate inside the cell. Eventually, both P and T approached constant levels, indicating that the system was reaching equilibrium stage and hydrate formation finished. 


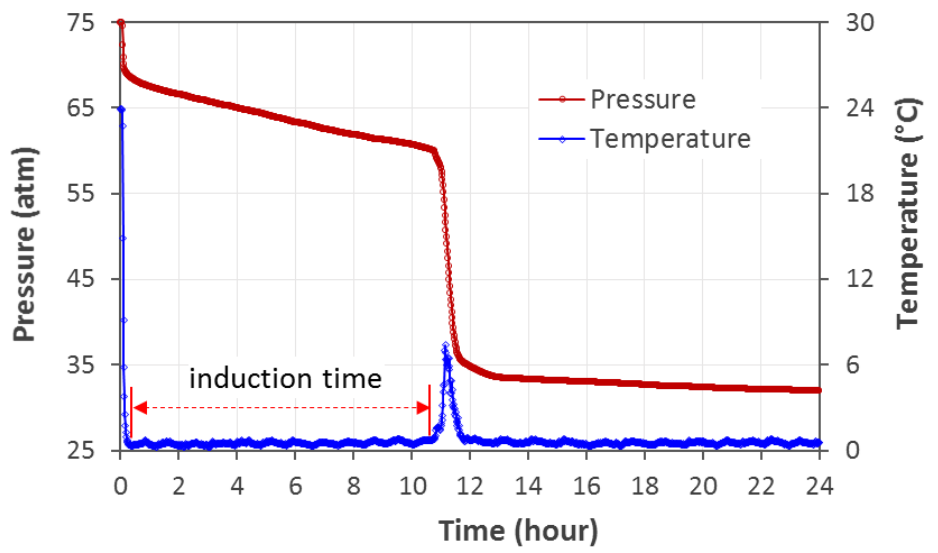

Figure 3. Typical P-T profile showing the change in pressure and temperature versus time during gas hydrate formation. The experiment was performed in an isobaric system. The initial pressure was $75 \mathrm{~atm}(7.5 \mathrm{MPa})$ and the target temperature was set to $0.5^{\circ} \mathrm{C}$.

Induction time: In gas hydrate formation, induction time represents the nucleation of gas hydrate ${ }^{53}$. It is theoretically defined as the period from the time when the T-P of a hydrating system reach to hydrate-forming condition till the time when the first hydrate crystal surpasses its critical size. At a molecular scale, it is the time needed for gas to dissolve into water to produce a supersaturated solution and for water structure to rearrange itself to accommodate the gas and form initial hydrate crystals. However, it is difficult to determine induction time through this theoretical concept. Alternatively, the induction time can be practically described by the period between the point when initial cooling completed and the point when the onset of hydrate growth is clearly observed, which is visually illustrated in Figure 3.

Figure 4 presents the induction time of methane hydrate formation versus the concentration of SDS. It is noted that, at some important concentrations, the experiments were repeated three to five times to determine the standard deviation and the reproducibility of the data. The obtained data on Figure 4 show that the average induction time of methane hydrate formation in neat water was 7.35 (hour). When the concentration of SDS was increased, the induction time increased first and then leveled off at 20.44 (hour) at $C_{S D S}=0.3 \mathrm{mM}$. Beyond this concentration, the induction time decreased continually with the increase of the concentration of SDS. In particular, the concentration of $5 \mathrm{mM}$ SDS gave an induction time of 0.23 hour, giving a 32-fold reduction compared to the case of pure water. 


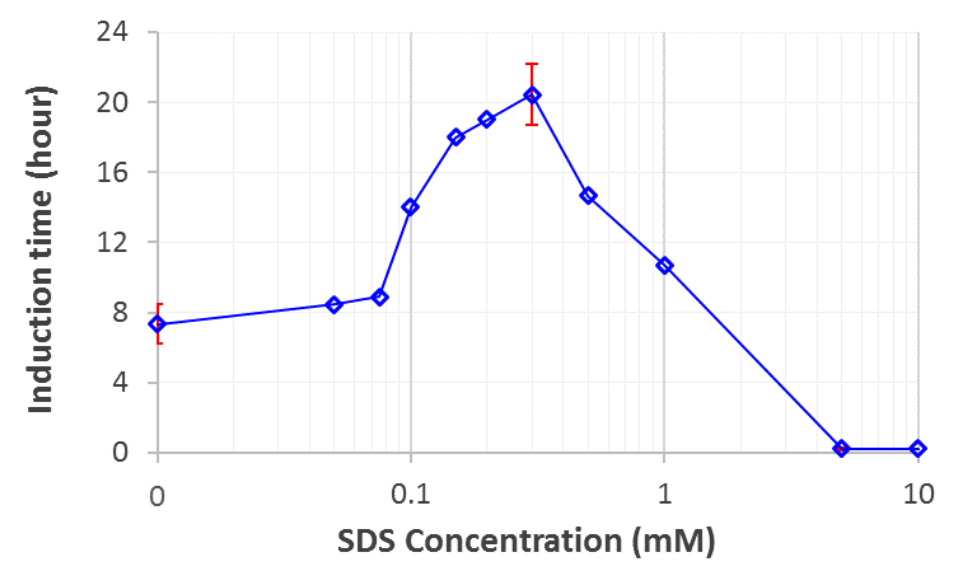

Figure 4. Induction time of methane hydrate formation in SDS solution of different concentrations. The data show an extraordinary inhibition observed at the concentration around $0.3 \mathrm{mM}$, along with a well-known promotion of concentrated SDS solution.

Gas uptake: In complement with the data about induction time, Figure 5 presents the normalised methane uptake by the hydrate as a function of time and SDS concentration. On the figure, a blue bay between $0.1 \mathrm{mM}$ and $0.5 \mathrm{mM}$ indicates a low gas uptake contour, while the red displays a region with high gas uptake. For example, at the SDS concentration of $0.3 \mathrm{mM}$, the color profile remains blue after 20 hours, indicating that gas uptake was still insignificant at that time. In contrast, at $5 \mathrm{mM}$, the color profile becomes red after a very short time, showing that high methane uptake was achieved shortly after the experiment started. Nonetheless, it is seeing that the accumulative gas uptake after 24 hours is almost similar for every concentration of SDS (and being around 0.11 mole $\mathrm{CH}_{4}$ per mole water). This observation indicates that although the induction time was strongly affected, the final methane uptake was not affected significantly. This is because, as per our observation, the water in the reactor was fully consumed after 24 hours and, hence, the methane uptake was terminated due to the shortage of water. 


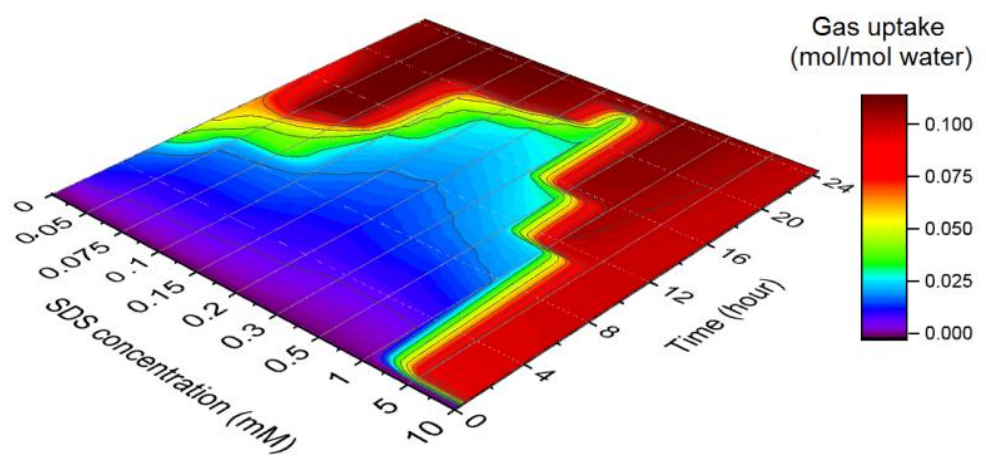

Figure 5. Time-dependent methane uptake by hydrate in SDS solution of different concentrations.

From the results about induction time and methane uptake, it is being shown that the dilute solution of SDS (especially at $0.3 \mathrm{mM}$ ) induces a strong inhibition of the formation of methane hydrate. In high concentration regime, SDS becomes an effective promoter. Although the promotion of gas hydrate formation by SDS is well known in the literature, the strong inhibition found in dilute SDS solution is extraordinary and fundamentally important. It implies that the affecting mechanism of SDS on gas hydrate formation needs to be carefully reconsidered. In the following sections, we used spectroscopic and simulation means to elucidate the underlying mechanism of this peculiar observation.

\subsection{Effect of SDS on interfacial water structure (SFG spectra)}

We employed Sum Frequency Generation vibrational spectroscopy (SFG) to study water structure at methane-water interfaces in the search for the origin of the inhibition observed in dilute SDS solution. SFG spectra are interface-specific and have been widely used for interface research, even though it was not used in gas hydrate-related studies.

Figure 6a shows a typical SFG spectrum of methane-SDS solution interface, recorded in the $3000-3600 \mathrm{~cm}^{-1}$ region. This region is of particular interest in this work as it is characteristic to the hydrogen-bonded water network ${ }^{54-55}$. On the figure, the experimental (measured) SFG data is shown in red circles, and the fitting of experimental data with modeled SFG is shown in solid black line. The green and blue curves, respectively, show the intensities of the two component peaks which constitute the spectral feature of this region. The center and the intensity 
of each peak were determined via fitting experimental data with SFG modules. The details of the fitting are presented in Supporting Information.
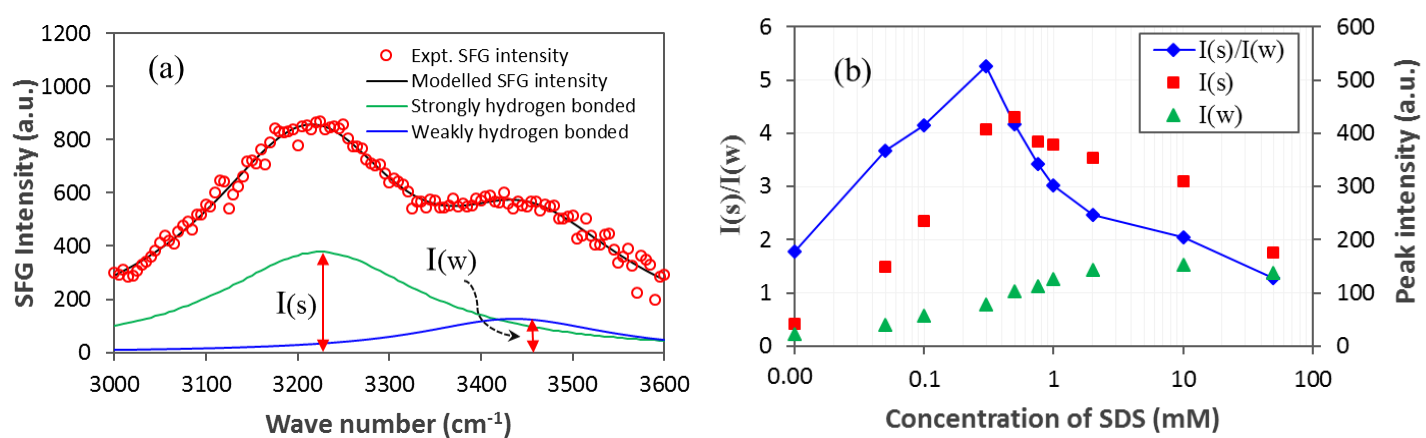

Figure 6. The data from SFG spectra. (a): a typical of experimental SFG spectra and its fitting components, and (b): the intensity of each component peak and the ratio between them as a function of SDS concentration. From graph (b), a strong ordering of water at the surface of 0.3 mM SDS solution is evidenced.

In Figure 6a, two broad peaks centered at 3220 and $3445 \mathrm{~cm}^{-1}$ are clearly identified. Although the configurational assignment of these peaks remains controversial ${ }^{55-60}$, it is factual that strongly-hydrogen-bonded $\mathrm{OHs}$ and weakly-hydrogen-bonded OHs, respectively, vibrate at lower and higher frequencies 55,61 . In accord with this general principle, the first peak centered at $3220 \mathrm{~cm}^{-1}$ is attributed to strongly hydrogen bonded $\mathrm{OHs}$ and, thus, manifests an ordered (oriented) configuration of water network ${ }^{62-63}$. Likewise, the second peak centered at $3445 \mathrm{~cm}^{-1}$ is assigned to weakly hydrogen bonded $\mathrm{OHs}$ and, therefore, is an indicator of a disordered configuration of water ${ }^{63-65}$. It should be, however, noted that the ordered configuration does not mean the water network is more tetrahedrally coordinated. Instead, it just means an enhancement in the intermolecular interaction between water molecules, which point will be clarified through this paper. Also in Figure 6a, the symbols $\mathrm{I}(\mathrm{s})$ and $\mathrm{I}(\mathrm{w})$ denote the intensity of the two peaks. As per the above assignments, the $\mathrm{I}(\mathrm{s}) / \mathrm{I}(\mathrm{w})$ ratio can be used as a measure of the ordering and hydrogen bond strength of the interfacial water ${ }^{66}$. For this reason, both the $\mathrm{I}(\mathrm{s}) / \mathrm{I}(\mathrm{w})$ ratio and its individual components are shown in Figure $6 \mathrm{~b}$ as a function of the concentration of SDS. From Figure 6b, two opposite patterns on the evolution of $\mathrm{I}(\mathrm{s}) / \mathrm{I}(\mathrm{w})$ are observed. In sub-millimolar regime, the ratio increases rapidly with the increase in SDS concentration and this increasing trend progresses until the concentration reaches $0.3 \mathrm{mM}$. From this concentration, the $\mathrm{I}(\mathrm{s}) / \mathrm{I}(\mathrm{w})$ 
turns to decreases continually with the concentration further increasing. Structurally, these spectral results reveal different effects of SDS on water structure at the interface. On one hand, the presence of SDS of sub-millimolar concentration results in an enhancement in water ordering and hydrogen bonds at the methane-solution interface. On the other hand, high concentrations of SDS lead to a depression of interfacial water followed by a weakening of hydrogen bonds.

\subsection{Simulation results}

Figure 7a shows an example of simulation results from System A. In this system, the total concentration of SDS in simulation box is $C_{t}=0.095 \mathrm{M}$. On the graph, the blue circles show the computed density of water and the blue line shows the fitting of the data using tanh function $\rho_{H_{2} O}=a \times\{1+\tanh [b \times(\Delta z-c)]\}$, in which $a=27.50, b=0.50$ and $c=0$. The origin of the horizontal axis $(\Delta z=0)$ indicates the position of Gibbs dividing surface (GDS). The left side of the GDS $(\Delta z<0)$ indicates the gas phase, and the right side $(\Delta z>0)$ indicates the liquid phase. The red squares show the density profile of dodecyl sulfate anions (denoted as $\mathrm{DS}^{-}$). From this density profile, a strong surface adsorption of $\mathrm{DS}^{-}$is shown by the enrichment of this anion in the interfacial region. Also on Figure 7a, the green squares indicate the orientational parameter, $\langle\cos \varphi\rangle$ calculated from Equation (1). By definition, this parameter provides a measure of water alignment at methane-solution interface and, therefore, is of central interest in this discussion. Evidently, the value of $\langle\cos \varphi\rangle$ varies greatly along the surface normal. It is well negative (namely -0.4) in the gas phase region near Gibbs dividing surface (GDS) which feature indicates that vaporised water molecules tend to point their hydrogen atoms towards gas phase. Similarly, the negative $\langle\cos \varphi\rangle$ (namely -0.1) in the sub-surface region indicates that the local water molecules are aligned into a configuration so that the molecular dipoles tend to point towards the bulk liquid (or hydrogen atoms pointing towards the interface). At a distance well far from the GDS, $\langle\cos \varphi\rangle$ approaches to zero which implies that the bulk condition has been reached. For convenience in later discussion, we define the interfacial depth as the region spreading from GDS to somewhere in the liquid phase at which $\langle\cos \varphi\rangle$ neutralized. 

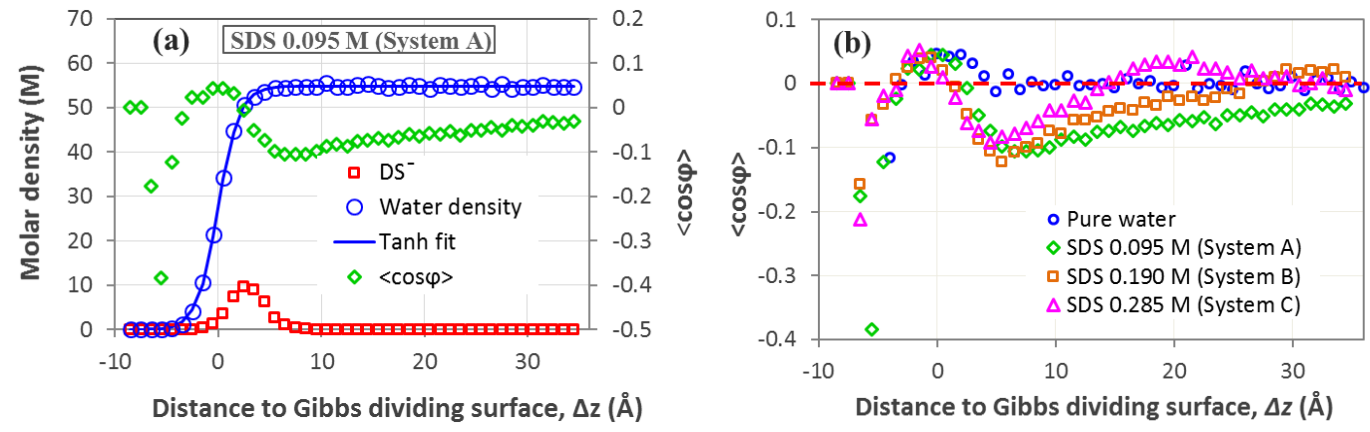

Figure 7. Properties of methane-water interface obtained from simulation. (a) A typical example of interfacial properties calculated from simulation and (b) the dependence of orientational parameter upon the total concentration of SDS in simulation box. The total concentration is defined as the molar amount of SDS divided by the volume of the aqueous phase in simulation box (in liter).

Figure $7 \mathrm{~b}$ shows the orientational parameter $\langle\cos \varphi\rangle$ of water in different simulation systems. First, it should be noted that although the value of $\langle\cos \varphi\rangle$ in the gas phase $(\Delta z<0)$ looks significant, it does not convey any valuable information for this work. The reason is that the gas phase just accounts for a few vaporised water molecules whereas this work is focusing on the liquid water underneath the interface. Therefore, the gas phase region in Figure $7 \mathrm{~b}$ is disregarded hereafter. Instead, the sub-surface region involving a layer of water underneath Gibbs dividing surface (GDS) is focused on. From Figure 7b, it is shown that pure water (blue circles) has neutral $\langle\cos \varphi\rangle$ in the sub-surface region, indicating that the local water does not have any orientational preference. In contrast, all SDS solutions display a significantly negative $\langle\cos \varphi\rangle$ in this region, which, by definition, indicates an aligned water layer underneath the GDS. Moreover, the interfacial depth decreases from System A $(\approx 35 \AA)$ to Systems $B(\approx 25 \AA)$ to System $C(\approx 15 \AA)$, suggesting that the extent of water alignment decreases in the same sequence. In fact, the fluctuation of $\langle\cos \varphi\rangle$ in System $\mathrm{C}$ may infer some perturbation to the alignment of water in this system.

These simulation results can be elucidated based on the distribution of $\mathrm{DS}^{-}$anions. First, the driving force of the water alignment is straightforward to understand. The surface adsorption of $\mathrm{DS}^{-}$anions also carries charges to the interface. The charged interface then induces an interfacial electrostatics field (IEF) which interacts with water dipoles and aligns them into a fashion that the water dipole vectors are favored to point towards the bulk water. Such alignment 
results in a negative $\langle\cos \varphi\rangle$ as shown on Figure 7b. However, the alignment of water is perturbed by the dynamics of water itself and, especially, the big $\mathrm{DS}^{-}$ions present in the subsurface region. In particular, all of the $\mathrm{DS}^{-}$anions in System A were adsorbed onto methanewater interface, leaving the concentration of $\mathrm{DS}^{-}$in the bulk being negligibly small (see $\mathrm{DS}^{-}$ profile on Figure 7a). The lack of $\mathrm{DS}^{-}$anion in sub-surface region gave no disturbance of water alignment, so that the water alignment was strongest in System A. In contrast, in Systems B and $\mathrm{C}$, simulation results (Supporting Information) show that only one portion of the initially inserted $\mathrm{DS}^{-}$anions was adsorbed onto methane-water interface while the remaining part resided in the bulk. Especially, the formation of a stable micelle was observed in the bulk of system $\mathrm{C}$ (Supporting Information). The presence of bulk-residing $\mathrm{SD}^{-}$anions, and especially the micelle, disturbed the alignment of water in Systems B and C, leading to a weakening of water alignment in these systems, as shown in Figure 7b. Because of this discussion, the condition of System A can be assigned to the sub-millimolar concentration in our experiments and the Systems B and C can reflect the high-concentration regime.

\subsection{Effect of mixture of SDS and TBAB}

In this section, we show how the effect of a mixture of SDS and TBAB is different from the individual effect of each surfactant. In our recent work ${ }^{37}$, we showed that TBAB (tetra-nbutylammonium bromide) displayed a strong inhibition of carbon dioxide hydrate formation when it was used at the concentration of $3.6 \mathrm{mM}$. Thus, we used this concentration for our aim here. On Figure 8, the induction time of methane hydrate formation is shown along with the corresponding $\mathrm{I}(\mathrm{s}) / \mathrm{I}(\mathrm{w})$, for neat water, each single surfactant and mixed surfactants. The average induction time in neat water and $0.3 \mathrm{mM}$ SDS solution was 7.35 (hour) and 20.44 (hour), respectively. In $3.6 \mathrm{mM}$ TBAB solution, no sign of methane hydrate growth was observed after 24 hours of experiments. Even though, the induction time for this system was assigned to be 24 hours for plotting. Interestingly, the average induction time of methane hydrate formation in a mixture of $0.3 \mathrm{mM}$ SDS and $3.6 \mathrm{mM}$ TBAB was only 2.27 (hour), being much shorter than those in the solution of each surfactant. In the meantime, the value of $I(s) / I(w)$ was smaller in the mixture than in the solutions of each surfactant. This result demonstrates that although both $0.3 \mathrm{mM}$ SDS solution and $3.6 \mathrm{mM}$ TBAB are strong inhibitors individually, the mixed solution of $0.3 \mathrm{mM}$ SDS and $3.6 \mathrm{mM}$ TBAB is an effective promoter. 


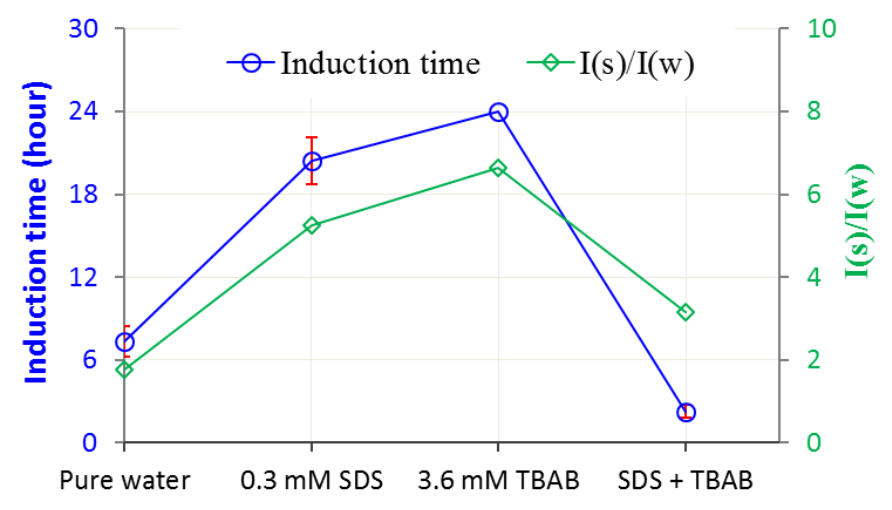

Figure 8. The effect of mixing surfactants on the induction time of gas hydrate formation and interfacial water alignment.

\section{GENERAL DISCUSSIONS}

\subsection{Correlation between water structure and hydrate kinetics}

Using the magnitude of $\mathrm{I}(\mathrm{s}) / \mathrm{I}(\mathrm{w})$ in SFG spectra as an indicator of water ordering at a gaswater interface ${ }^{64-65}$, we have identified two distinct configurations of interfacial water respectively at the surface of dilute and concentrated SDS solution. In low concentration, the presence of SDS induces an enhanced ordering of water at the solution surface and the greatest extent of water ordering is achieved at $C_{S D S}=0.3 \mathrm{mM}$. In higher concentration, a depression of water structure followed by a weakening of hydrogen bond network is evidenced through the down turn of $\mathrm{I}(\mathrm{s}) / \mathrm{I}(\mathrm{w})$. Interestingly, this trend can be explained by simulation results. First, our simulations indicate that the ordering of water originates from the alignment of interfacial water under the effect of charged $\mathrm{DS}^{-}$absorbed on the interface. Secondly, the absence of $\mathrm{DS}^{-}$anions in the sub-surface of dilute SDS solution gives no disturbance of water aligning process, which could give a reasonable explanation to the strongest water ordering observed spectroscopically at $C_{S D S}=0.3 \mathrm{mM}$. Thirdly, the increasing presence of $\mathrm{DS}^{-}$anions and, especially, the micelles in the sub-surface of concentrated SDS solution perturbs the alignment of interfacial water (as observed in Systems C of the simulation), which could ultimately be responsible for the weakening of water ordering observed spectroscopically in the higher concentration. 
Interestingly, the change in the structure of interfacial water has a strong correlation with the measured methane hydrate kinetics. Using the term $\mathrm{I}(\mathrm{s}) / \mathrm{I}(\mathrm{w})$ to express the water alignment and the induction time to express methane hydrate kinetics, an intrinsic correlation between structural and kinetic properties is shown in Figure 9. Evidently, the strongest interfacial water alignment is observed together with the longest induction time at $C_{S D S}=0.3 \mathrm{mM}$. This observation should not be considered as a coincidence since a similar matching was also observed at $C_{T B A B}=3.6 \mathrm{mM}$ for $\mathrm{TBAB}$ and $\mathrm{CO}_{2}$ hydrate in our recent work ${ }^{37}$. Moreover, in Figure 8 , it is shown that the correlation (stronger alignment-longer induction time) is also satisfied for methane hydrate formation in $3.6 \mathrm{mM}$ TBAB solution. The evidence becomes more strengthened when it is being shown that a mixed solution of $0.3 \mathrm{mM}$ SDS and $3.6 \mathrm{mM}$ TBAB displays both a shortening of the induction time and a weakening of water alignment (see Figure 8). The weakening of the water alignment in this mixture is expected because the co-adsorption of two oppositely charged surfactants can lead to the neutralization of surface charge. Based on these results, we conclude that the alignment of water underneath surface adsorption of surfactants is the origin of the extraordinary inhibition of gas hydrate formation in dilute surfactant solutions.

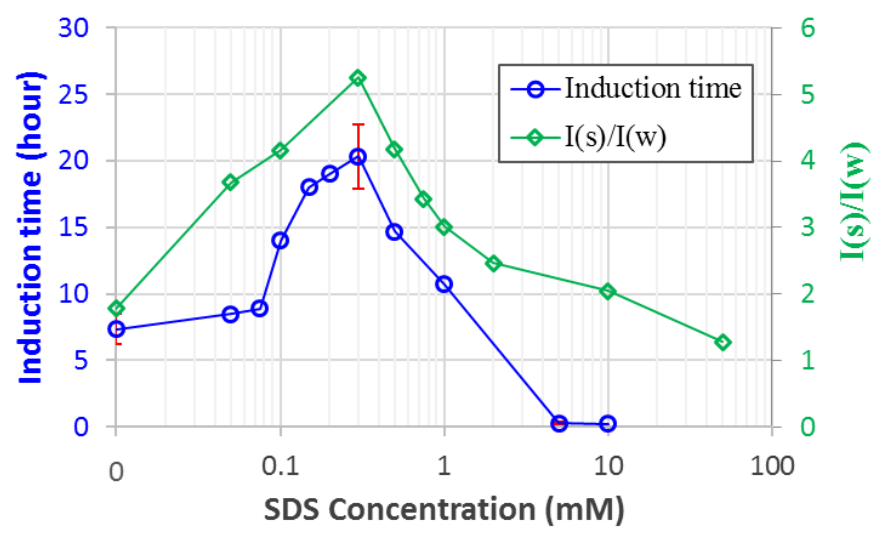

Figure 9. The correlation between methane hydrate kinetics and interfacial water alignment in SDS solution. The data show that a higher degree of interfacial water alignment results in slower hydrate formation kinetics. 


\subsection{Interfacial water alignment versus gas hydrate formability}

We have evidently shown the water alignment at gas-solution interfaces being an inhibitor of gas hydrate formation. This is a freshly new finding. Here we discuss the physics underlying this. First, it is worth remembering that the formation of gas hydrate usually initiates at gas-water interfaces ${ }^{39-41}$ where the gas solubility is locally high enough to meet the required supersaturated condition for triggering the clathrate nucleation ${ }^{1}$. During the event of the nucleation, local water molecules (in the interfacial region) have to rearrange to accommodate the gas (methane) and comfort themselves with the presence of the guest. This process is also known as the hydrophobic hydration of gas. In some certain conditions, the resulting hydrophobic hydration shells can further develop into hydrate cages, and then the cages build up hydrate crystals ${ }^{1,42,67}$.

Hence, the initially aligned water at the gas-water interface would create an unfavorable condition for the nucleation process. This is because, the nucleation of gas hydrates, as discussed above, requires a structural rearrangement of water molecules at the interface. Therefore, when interfacial water is aligned by an external field (e.g. the interfacial electrostatic field, IEF), the structural rearrangement becomes unfavorable as the driving force of nucleation has to surpass the electrostatic interaction between water dipoles and the field. The driving force of hydrate nucleation tends to rearrange water into clathrate-like conformation whereas the IEF inclines the water into aligned. Consequently, the nucleation of gas hydrate is hindered. Figure 10 illustrates the concept discussed here. Figure 10a shows the hydration of a methane molecule in ordinary water. In this snapshot, a water shell surrounding methane molecule with a hydrogen bond network is identified. This hydration shell can develop into a hydrate cage by transforming its structure and exchanging water molecules with the bulk. In contract, Figure 10b indicates an incomplete hydration of methane in the aligned water structure in which the construction of the hydration shell is hindered due to the orientation of water molecules. This mechanism gives rise to the inhibition of gas hydrate formation in dilute SDS and TBAB solutions, as we have shown. Moreover, the inhibition though this mechanism is on the nucleation stage, explaining why the induction time is strongly affected by the surfactant. 

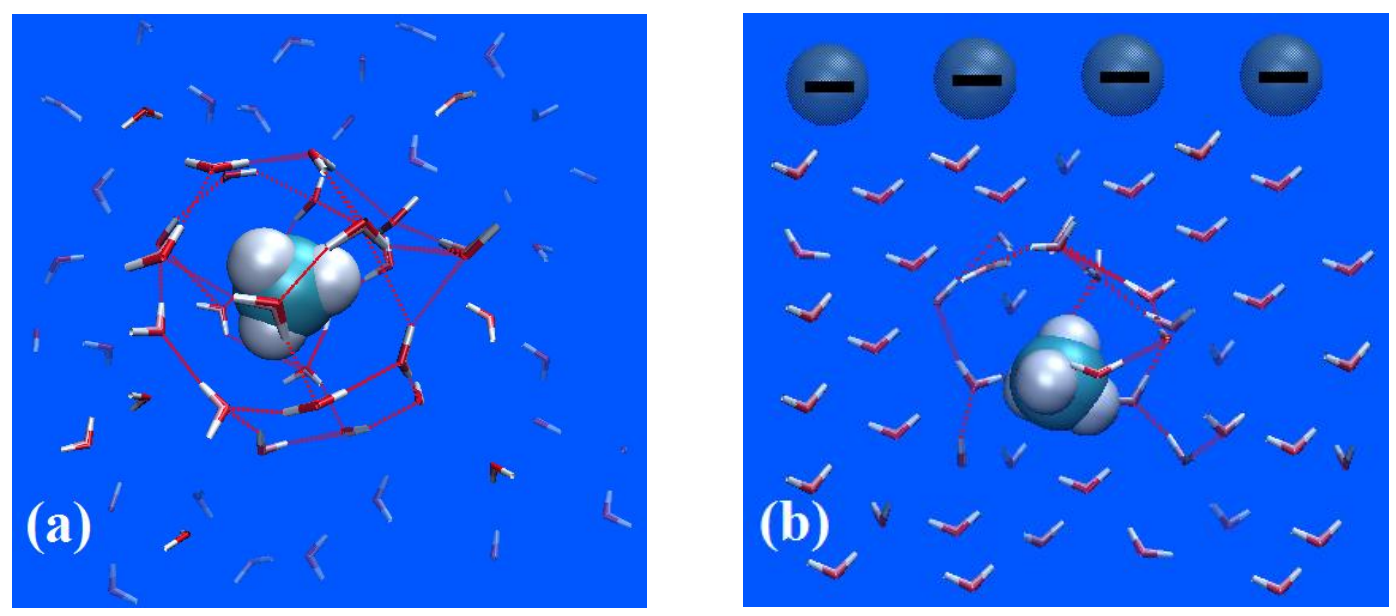

Figure 10. The hydration of methane in ordinary water (a) and aligned water (b). The hydrogen bonds between water molecules on hydration shells are shown. The dark spheres with the negative sign represnt the head group of surfactant. The surfactant-induced alignment of water results in an unfavourable condition for the hydration of methane (b).

Finally, it is noted that the promotion of methane hydrate formation in concentrated SDS solution is well-known in literature and, thus, is not focussed on in this work. However, it is worth to show that such promotion does not conflict with the mechanism proposed above. Indeed, the weakening of water alignment in concentrated SDS solution is an expected observation, given the promotion being known in this concentration range. In the meantime, the presence of $\mathrm{DS}^{-}$anions and its micelles in the sub-surface region can enhance the local solubility of methane ${ }^{26,38}$.

\section{CONCLUSION}

We have employed a synergic combination of kinetic measurements, surface-specific SFG spectroscopy and molecular dynamics (MD) simulation to study, at a molecular scale, the effect of sodium dodecyl sulfate (SDS) on the formability of methane gas hydrate. Kinetic results show an extraordinary inhibition of the methane hydrate formation in sub-millimolar SDS solution (especially at $C_{S D S}=0.3 \mathrm{mM}$ ), along with a well-known promotion effect in high concentration regime. Moreover, we have established an interesting evidence that the mixed solution of 0.3 $\mathrm{mM}$ SDS and $3.6 \mathrm{mM}$ TBAB provides a good promoter despite the fact that the $3.6 \mathrm{mM}$ TBAB solution itself is also an inhibitor. The results from SFG measurements and MD simulations 
show that the alignment of water underneath surface adsorption of surfactant ions gives rise to the inhibition effect. We have discussed this novel finding using the concept of energy barriers of the transformation of water structure at the interface during the nucleation of gas hydrates. The vanishing of the inhibition effect in the mixed surfactants solution is explained through the weakening of interfacial water alignment due to the neutralization of interface charge. This work has shed light into a universal understanding of the effect of SDS and TBAB on gas hydrate formation.

\section{Acknowledgement}

The authors gratefully acknowledge the Australian Government for the Australian Awards Scholarship (AusAID PhD Scholarship) for Ngoc N. Nguyen. The U.S. Department of Energy, Office of Science, Office of Basic Energy Sciences, Division of Chemical Sciences, Geosciences and Biosciences funded the work performed by Liem X. Dang.

\section{References}

(1) Sloan, E. D.; Koh, C. A. Clathrate Hydrates of Natural Gases, Third Edition; Crc PressTaylor \& Francis Group: Boca Raton, 2008; Vol. 119, p 1-701.

(2) Sloan, E. D. Fundamental Principles and Applications of Natural Gas Hydrates. Nature 2003, 426, 353-359.

(3) Hammerschmidt, E. G. Formation of Gas Hydrates in Natural Gas Transmission Lines. Industrial and Engineering Chemistry 1934, 26, 851-855.

(4) Giavarini, C.; Hester, K., Gas Hydrates: Immense Energy Potential and Environmental Challenges. Springer London: London, 2011; pp. 1-175.

(5) Boswell, R.; Collett, T. S. Current Perspectives on Gas Hydrate Resources. Energy \& Environmental Science 2011, 4, 1206-1215.

(6) Li, X. S.; Xu, C. G.; Zhang, Y.; Ruan, X. K.; Li, G.; Wang, Y. Investigation into Gas Production from Natural Gas Hydrate: A Review. Appl. Energy 2016, 172, 286-322.

(7) Pang, W. X.; Xu, W. Y.; Sun, C. Y.; Zhang, C. L.; Chen, G. J. Methane Hydrate Dissociation Experiment in a Middle-Sized Quiescent Reactor Using Thermal Method. Fuel 2009, 88, 497-503.

(8) Yuan, Q.; Sun, C. Y.; Yang, X.; Ma, P. C.; Ma, Z. W.; Liu, B.; Ma, Q. L.; Yang, L. Y.; Chen, G. J. Recovery of Methane from Hydrate Reservoir with Gaseous Carbon Dioxide Using a Three-Dimensional Middle-Size Reactor. Energy 2012, 40, 47-58. 
(9) Wang, W. X.; Bray, C. L.; Adams, D. J.; Cooper, A. I. Methane Storage in Dry Water Gas Hydrates. Journal of the American Chemical Society 2008, 130, 11608-+.

(10) Carter, B. O.; Wang, W. X.; Adams, D. J.; Cooper, A. I. Gas Storage in "Dry Water" and "Dry Gel" Clathrates. Langmuir 2010, 26, 3186-3193.

(11) Casco, M. E., et al. Methane Hydrate Formation in Confined Nanospace Can Surpass Nature. Nature Communications 2015, 6.

(12) Veluswamy, H. P.; Wong, A. J. H.; Babu, P.; Kumar, R.; Kulprathipanja, S.; Rangsunvigit, P.; Linga, P. Rapid Methane Hydrate Formation to Develop a Cost Effective Large Scale Energy Storage System. Chemical Engineering Journal 2016, 290, 161-173.

(13) Li, X. S.; Xu, C. G.; Chen, Z. Y.; Wu, H. J. Tetra-N-Butyl Ammonium Bromide SemiClathrate Hydrate Process for Post-Combustion Capture of Carbon Dioxide in the Presence of Dodecyl Trimethyl Ammonium Chloride. Energy 2010, 35, 3902-3908.

(14) Lee, H. J.; Lee, J. D.; Linga, P.; Englezos, P.; Kim, Y. S.; Lee, M. S.; Kim, Y. D. Gas Hydrate Formation Process for Pre-Combustion Capture of Carbon Dioxide. Energy 2010, 35, 2729-2733.

(15) Park, S.; Lee, S.; Lee, Y.; Lee, Y.; Seo, Y. Hydrate-Based Pre-Combustion Capture of Carbon Dioxide in the Presence of a Thermodynamic Promoter and Porous Silica Gels. International Journal of Greenhouse Gas Control 2013, 14, 193-199.

(16) Xu, C. G.; Chen, Z. Y.; Cai, J.; Li, X. S. Study on Pilot-Scale Co2 Separation from Flue Gas by the Hydrate Method. Energy \& Fuels 2014, 28, 1242-1248.

(17) Babu, P.; Chin, W. I.; Kumar, R.; Linga, P. Systematic Evaluation of Tetra-N-Butyl Ammonium Bromide (Tbab) for Carbon Dioxide Capture Employing the Clathrate Process. Industrial \& Engineering Chemistry Research 2014, 53, 4878-4887.

(18) Babu, P.; Kumar, R.; Linga, P. A New Porous Material to Enhance the Kinetics of Clathrate Process: Application to Precombustion Carbon Dioxide Capture. Environmental Science \& Technology 2013, 47, 13191-13198.

(19) Li, X. S.; Xia, Z. M.; Chen, Z. Y.; Wu, H. J. Precombustion Capture of Carbon Dioxide and Hydrogen with a One-Stage Hydrate/Membrane Process in the Presence of Tetra-NButylammonium Bromide (Tbab). Energy \& Fuels 2011, 25, 1302-1309.

(20) Sun, C.-Y.; Ma, C.-F.; Chen, G.-J.; Zhang, S.-X. Experimental and Simulation of Single Equilibrium Stage Separation of (Methane + Hydrogen) Mixtures Via Forming Hydrate. Fluid Phase Equilibria 2007, 261, 85-91.

(21) Tohidi, B.; Yang, J. H.; Salehabadi, M.; Anderson, R.; Chapoy, A. Co2 Hydrates Could Provide Secondary Safety Factor in Subsurface Sequestration of Co2. Environmental Science \& Technology 2010, 44, 1509-1514.

(22) Koide, H.; Shindo, Y.; Tazaki, Y.; Iijima, M.; Ito, K.; Kimura, N.; Omata, K. Deep SubSeabed Disposal of $\mathrm{Co} 2$ - the Most Protective Storage. Energy Conversion and Management 1997, 38, S253-S258.

(23) Qanbari, F.; Pooladi-Darvish, M.; Tabatabaie, S. H.; Gerami, S. Co2 Disposal as Hydrate in Ocean Sediments. Journal of Natural Gas Science and Engineering 2012, 8, 139-149. 
(24) Yuan, Q.; Wang, X. H.; Dandekar, A.; Sun, C. Y.; Li, Q. P.; Ma, Z. W.; Liu, B.; Chen, G. J. Replacement of Methane from Hydrates in Porous Sediments with Co2-in-Water Emulsions. Ind. Eng. Chem. Res. 2014, 53, 12476-12484.

(25) Kumar, A.; Bhattacharjee, G.; Kulkarni, B. D.; Kumar, R. Role of Surfactants in Promoting Gas Hydrate Formation. Industrial \& Engineering Chemistry Research 2015, 54, 12217-12232.

(26) Zhong, Y.; Rogers, R. E. Surfactant Effects on Gas Hydrate Formation. Chemical Engineering Science 2000, 55, 4175-4187.

(27) Torre, J. P.; Dicharry, C.; Ricaurte, M.; Daniel-David, D.; Broseta, D. Co2 Capture by Hydrate Formation in Quiescent Conditions: In Search of Efficient Kinetic Additives. 10th International Conference on Greenhouse Gas Control Technologies 2011, 4, 621-628.

(28) Ando, N.; Kuwabara, Y.; Mori, Y. H. Surfactant Effects on Hydrate Formation in an Unstirred Gas/Liquid System: An Experimental Study Using Methane and MicelleForming Surfactants. Chemical Engineering Science 2012, 73, 79-85.

(29) Babu, P.; Linga, P.; Kumar, R.; Englezos, P. A Review of the Hydrate Based Gas Separation (Hbgs) Process For carbon Dioxide Pre-Combustion Capture. Energy 2015, 85, 261-279.

(30) Lin, W.; Chen, G. J.; Sun, C. Y.; Guo, X. Q.; Wu, Z. K.; Liang, M. Y.; Chen, L. T.; Yang, L. Y. Effect of Surfactant on the Formation and Dissociation Kinetic Behavior of Methane Hydrate. Chemical Engineering Science 2004, 59, 4449-4455.

(31) Zhang, L. W.; Chen, G. J.; Sun, C. Y.; Fan, S. S.; Ding, Y. M.; Wang, X. L.; Yang, L. Y. The Partition Coefficients of Ethylene between Hydrate and Vapor for Methane + Ethylene + Water and Methane + Ethylene + Sds + Water Systems. Chemical Engineering Science 2005, 60, 5356-5362.

(32) Hong, S. Y.; Jim, J. I.; Kim, J. H.; Lee, J. D. Kinetic Studies on Methane Hydrate Formation in the Presence of Kinetic Inhibitor Via in Situ Raman Spectroscopy. Energy Fuels 2012, 26, 7045-7050.

(33) Heidaryan, E.; Salarabadi, A.; Moghadasi, J.; Dourbash, A. A New High Performance Gas Hydrate Inhibitor. J. Nat. Gas Chem. 2010, 19, 323-326.

(34) Nazari, K.; Moradi, M. R.; Ahmadi, A. N. Kinetic Modeling of Methane Hydrate Formation in the Presence of Low-Dosage Water-Soluble Ionic Liquids. Chem. Eng. Technol. 2013, 36, 1915-1923.

(35) Jarrahian, A.; Heidaryan, E. Natural Gas Hydrate Promotion Capabilities of Toluene Sulfonic Acid Isomers. Pol. J. Chem. Technol. 2014, 16, 97-102.

(36) Sowa, B.; Maeda, N. Probability Distributions of Natural Gas Hydrate Formation in Sodium Dodecyl Sulfate Aqueous Solutions. Energy \& Fuels 2015, 29, 5692-5700.

(37) Nguyen, N. N.; Nguyen, A. V.; Nguyen, K. T.; Rintoul, L.; Dang, L. X. Unexpected Inhibition of $\mathrm{Co} 2$ Gas Hydrate Formation in Dilute Tbab Solutions and the Critical Role of Interfacial Water Structure. Fuel 2016, 185, 517-523. 
(38) Rogers, R.; Zhang, G.; Dearman, J.; Woods, C. Investigations into Surfactant/Gas Hydrate Relationship. Journal of Petroleum Science and Engineering 2007, 56, 82-88.

(39) Taylor, C. J.; Miller, K. T.; Koh, C. A.; Sloan, E. D. Macroscopic Investigation of Hydrate Film Growth at the Hydrocarbon/Water Interface. Chemical Engineering Science 2007, 62, 6524-6533.

(40) Li, S. L.; Sun, C. Y.; Liu, B.; Li, Z. Y.; Chen, G. J.; Sum, A. K. New Observations and Insights into the Morphology and Growth Kinetics of Hydrate Films. Sci Rep 2014, 4, 6.

(41) Daniel-David, D.; Guerton, F.; Dicharry, C.; Torre, J. P.; Broseta, D. Hydrate Growth at the Interface between Water and Pure or Mixed Co2/Ch4 Gases: Influence of Pressure, Temperature, Gas Composition and Water-Soluble Surfactants. Chemical Engineering Science 2015, 132, 118-127.

(42) Nguyen, N. N.; Nguyen, A. V. The Dual Effect of Sodium Halides on the Formation of Methane Gas Hydrate. Fuel 2015, 156, 87-95.

(43) Nguyen, N. N.; Nguyen, A. V.; Steel, K. M.; Dang, L. X.; Galib, M. Interfacial Gas Enrichment at Hydrophobic Surfaces and the Origin of Promotion of Gas Hydrate Formation by Hydrophobic Solid Particles. The Journal of Physical Chemistry C 2017.

(44) Guo, B.; Ghalambor, A. Natural Gas Engineering Handbook (2nd Edition). Gulf Publishing Company.

(45) D.A. Case, V. B., J.T. Berryman, R.M. Betz, Q. Cai, D.S. Cerutti, T.E. Cheatham, III, T.A. Darden, R.E.; Duke, H. G., A.W. Goetz, S. Gusarov, N. Homeyer, P. Janowski, J. Kaus, I. Kolossváry, A. Kovalenko,; T.S. Lee, S. L., T. Luchko, R. Luo, B. Madej, K.M. Merz, F. Paesani, D.R. Roe, A. Roitberg, C. Sagui,; R. Salomon-Ferrer, G. S., C.L. Simmerling, W. Smith, J. Swails, R.C. Walker, J. Wang, R.M. Wolf, X.; Kollman, W. a. P. A. Amber 14. University of California, San Francisco 2014, 1-826.

(46) Case, D. A.; Cheatham, T. E.; Darden, T.; Gohlke, H.; Luo, R.; Merz, K. M.; Onufriev, A.; Simmerling, C.; Wang, B.; Woods, R. J. The Amber Biomolecular Simulation Programs. J. Comput. Chem. 2005, 26, 1668-1688.

(47) Horn, H. W.; Swope, W. C.; Pitera, J. W.; Madura, J. D.; Dick, T. J.; Hura, G. L.; HeadGordon, T. Development of an Improved Four-Site Water Model for Biomolecular Simulations: Tip4p-Ew. Journal of Chemical Physics 2004, 120, 9665-9678.

(48) Jorgensen, W. L.; Maxwell, D. S.; TiradoRives, J. Development and Testing of the Opls All-Atom Force Field on Conformational Energetics and Properties of Organic Liquids. Journal of the American Chemical Society 1996, 118, 11225-11236.

(49) Weiner, S. J.; Kollman, P. A.; Case, D. A.; Singh, U. C.; Ghio, C.; Alagona, G.; Profeta, S.; Weiner, P. A New Force-Field for Molecular Mechanical Simulation of Nucleic Acids and Proteins. Journal of the American Chemical Society 1984, 106, 765-784.

(50) Cornell, W. D.; Cieplak, P.; Bayly, C. I.; Gould, I. R.; Merz, K. M.; Ferguson, D. M.; Spellmeyer, D. C.; Fox, T.; Caldwell, J. W.; Kollman, P. A. A Second Generation Force Field for the Simulation of Proteins, Nucleic Acids, and Organic Molecules (Vol 117, Pg 5179, 1995). Journal of the American Chemical Society 1996, 118, 2309-2309. 
(51) Martinez, L.; Andrade, R.; Birgin, E. G.; Martinez, J. M. Packmol: A Package for Building Initial Configurations for Molecular Dynamics Simulations. J. Comput. Chem. 2009, 30, 2157-2164.

(52) Essmann, U.; Perera, L.; Berkowitz, M. L.; Darden, T.; Lee, H.; Pedersen, L. G. A Smooth Particle Mesh Ewald Method. Journal of Chemical Physics 1995, 103, 8577-8593.

(53) Christiansen, R. L.; Sloan, E. D. Mechanisms and Kinetics of Hydrate Formation. In International Conference on Natural Gas Hydrates, Sloan, E. D.; Happel, J.; Hnatow, M. A., Eds. New York Acad Sciences: New York, 1994; Vol. 715, pp 283-305.

(54) Vidal, F.; Tadjeddine, A. Sum-Frequency Generation Spectroscopy of Interfaces. Reports on Progress in Physics 2005, 68, 1095-1127.

(55) Perakis, F.; De Marco, L.; Shalit, A.; Tang, F. J.; Kann, Z. R.; Kuhne, T. D.; Torre, R.; Bonn, M.; Nagata, Y. Vibrational Spectroscopy and Dynamics of Water. Chemical Reviews 2016, 116, 7590-7607.

(56) Nihonyanagi, S.; Ishiyama, T.; Lee, T.; Yamaguchi, S.; Bonn, M.; Morita, A.; Tahara, T. Unified Molecular View of the Air/Water Interface Based on Experimental and Theoretical Chi((2)) Spectra of an Isotopically Diluted Water Surface. Journal of the American Chemical Society 2011, 133, 16875-16880.

(57) Sovago, M.; Campen, R. K.; Wurpel, G. W. H.; Muller, M.; Bakker, H. J.; Bonn, M. Vibrational Response of Hydrogen-Bonded Interfacial Water Is Dominated by Intramolecular Coupling. Physical Review Letters 2008, 100, 4.

(58) Tian, C. S.; Shen, Y. R. Comment on "Vibrational Response of Hydrogen-Bonded Interfacial Water Is Dominated by Intramolecular Coupling". Physical Review Letters 2008, 101,1 .

(59) Sovago, M.; Campen, R. K.; Wurpel, G. W. H.; Muller, M.; Bakker, H. J.; Bonn, M. Comment on "Vibrational Response of Hydrogen-Bonded Interfacial Water Is Dominated by Intramolecular Coupling" - Reply. Physical Review Letters 2008, 101, 1.

(60) Nihonyanagi, S.; Yamaguchi, S.; Tahara, T. Water Hydrogen Bond Structure near Highly Charged Interfaces Is Not Like Ice. Journal of the American Chemical Society 2010, 132, 6867-6869.

(61) Bakker, H. J. Femtosecond Mid-Infrared Spectroscopy of Liquid Water and Aqueous Solutions. In Spectroscopic Properties of Inorganic and Organometallic Compounds: Techniques, Materials and Applications, Vol 41, Yarwood, J.; Douthwaite, R.; Duckett, S. B., Eds. Royal Soc Chemistry: Cambridge, 2010; Vol. 41, pp 166-210.

(62) Shen, Y. R.; Ostroverkhov, V. Sum-Frequency Vibrational Spectroscopy on Water Interfaces: Polar Orientation of Water Molecules at Interfaces. Chemical Reviews 2006, 106, 1140-1154.

(63) Allen, H. C.; Casillas-Ituarte, N. N.; Sierra-Hernandez, M. R.; Chen, X. K.; Tang, C. Y. Shedding Light on Water Structure at Air-Aqueous Interfaces: Ions, Lipids, and Hydration. Physical Chemistry Chemical Physics 2009, 11, 5538-5549. 
(64) Richmond, G. L. Structure and Bonding of Molecules at Aqueous Surfaces. Annu. Rev. Phys. Chem. 2001, 52, 357-389.

(65) Gragson, D. E.; McCarty, B. M.; Richmond, G. L. Ordering of Interfacial Water Molecules at the Charged Air/Water Interface Observed by Vibrational Sum Frequency Generation. Journal of the American Chemical Society 1997, 119, 6144-6152.

(66) Liu, D. F.; Ma, G.; Levering, L. M.; Allen, H. C. Vibrational Spectroscopy of Aqueous Sodium Halide Solutions and Air-Liquid Interfaces: Observation of Increased Interfacial Depth. Journal of Physical Chemistry B 2004, 108, 2252-2260.

(67) Walsh, M. R.; Koh, C. A.; Sloan, E. D.; Sum, A. K.; Wu, D. T. Microsecond Simulations of Spontaneous Methane Hydrate Nucleation and Growth. Science 2009, 326, 1095-1098. 